\title{
Diaphragmatic Eventration
}

National Cancer Institute

\section{Source}

National Cancer Institute. Diaphragmatic Eventration. NCI Thesaurus. Code C98912.

A cong enital or acquired abnormality characterized by elevation of the hemidiaphragm. 